Received: 2016.01.31

Accepted: 2016.03.01

Published: 2016.10 .29

Authors' Contribution:

Study Design A

Data Collection B

Statistical Analysis C

Data Interpretation D

Manuscript Preparation E

Literature Search F

Funds Collection $G$

\title{
Gremlin-1 Concentrations Are Correlated with the Severity of Knee Osteoarthritis
}

BE 1,2 Jun Yi

AG 1,3 Qunhua Jin

CF 2 Bin Zhang

BD 2 Xinqiu Wu

BF 2 Dawei Ge
1 Medical College, Shandong University, Jinan, Shandong, P.R. China

2 Department of Orthopaedics, Shuyang Traditional Chinese Medicine Hospital,

Shuyang, Jiangsu, P.R. China

3 Department of Orthopaedics, General Hospital, Ningxia Medical University,

Yinchuan, Ningxia, P.R. China
Corresponding Author:

Source of support:
Qunhua Jin, e-mail: qunhjin@yeah.net Departmental sources

Background: Gremlin-1, a bone morphogenetic protein (BMP) antagonist, is up-regulated in osteoarthritis (OA). Therefore, we aim to evaluate the correlation between gremlin-1 concentrations and the onset and severity of OA.

Material/Methods: We performed this cross-sectional study in a population of 212 patients with knee OA and 125 healthy controls. Results: Patients with knee OA had higher serum gremlin-1 concentrations than healthy controls. Serum and synovial fluid (SF) gremlin-1 concentrations increased according to advanced Kellgren-Lawrence grading stages.

Conclusions: Serum and SF gremlin-1 concentrations are correlated with the onset and severity of knee OA.

MeSH Keywords: $\quad$ Bone Morphogenetic Proteins • Injury Severity Score • Osteoarthritis, Knee

Full-text PDF: http://www.medscimonit.com/abstract/index/idArt/897849

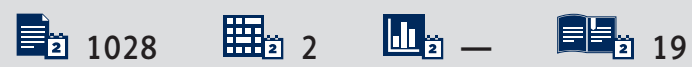




\section{Background}

Osteoarthritis (OA), a common joint disease, results in pain, stiffness, reduced motion, swelling, crepitus, and disability [1]. The progressive destruction of articular cartilage, joint-space narrowing, and subchondral sclerosis are the typical characteristics of OA [2]. The etiology and pathogenesis of OA remain to be unclear. Recent investigations showed that aging, inflammation, and obesity were correlated with OA pathogenesis $[3,4]$.

Bone morphogenetic protein (BMP), a member of transforming growth factor- $\beta$ (TGF- $\beta$ ) superfamily, is involved in bone formation and repair of bone and cartilage [5]. Gremlin-1, a BMP antagonist, has high binding ability with BMP-2 and BMP-4 [6]. The balance between gremlin-1 and BMP is correlated with chondrocyte hypertrophy and joint cartilage homeostasis [7] It has been reported that gremlin-1 was significantly up-regulated in osteoarthritic human chondrocytes [8]. We postulate that gremlin-1 may be a key regulator in $\mathrm{OA}$ onset.

The aim of this investigation is to determine the relation between gremlin-1 concentrations and the onset and severity of $O A$.

\section{Material and Methods}

\section{Patients}

This investigation was conducted in a population of 212 patients with knee OA and 125 healthy controls. These patients were diagnosed with knee OA using the criteria of the American College of Rheumatology [9]. The exclusive criteria were as follows: inflammatory knee disease, rheumatoid arthritis, malignant tumors, systemic diseases, autoimmune diseases, and corticosteroids treatment during the past three months. The control group was randomly selected from healthy volunteers who visited the general health check-up center of our hospital. They were confirmed with normal knee radiography and had no systemic disease. Informed written consent was obtained from all subjects and the institutional review board of the institution approved the protocol.

Disease severity was scored according to the Kellgren-Lawrence $(\mathrm{KL})$ grading system by 2 radiologists. Those with $\mathrm{KL}$ grade $\geq 2$ in at least 1 knee were considered to have radiographic knee OA. Healthy controls all have 2 knees with grade of 0 .

\section{Laboratory methods}

Synovial fluid (SF) was abstracted from the knee before hyaluronic acid therapy. SF was then centrifuged and stored at $-20^{\circ} \mathrm{C}$ before measurement. An enzyme-linked immunosorbent assay kit (R\&D Company) was utilized to measure serum and SF gremlin-1 concentrations.

\section{Statistical analysis}

The data were displayed as a form of means \pm SD or median (interquartile range). We then utilized chi-square tests, unpaired $t$ test, or Mann-Whitney $U$ test to assess the parameter variance of gender; age and body mass index (BMI); as well as gremlin-1 concentrations, respectively, between the cases and control groups. Serum and SF gremlin-1 concentration differences between knee OA subgroups were assessed using the Kruskal-Wallis test. Spearman correlation analysis and multinomial logistic regression analysis were utilized to show the correlation of gremlin-1 concentrations with $\mathrm{KL}$ grading. $P<0.05$ was considered statistically significant.

\section{Results}

\section{Clinical parameters of the case and control group}

There were no age, sex, or BMI differences between these 2 groups (Table 1).

\section{Serum gremlin-1 concentrations}

Higher serum gremlin-1 concentrations were shown in the case group than in the control group $(P<0.001$; Table 1$)$.

\section{Gremlin-1 concentrations correlated with KL grades}

Serum and SF gremlin-1 concentrations increased as KL grading stages advanced (Table 2). Spearman correlation analysis presented a positive correlation between serum and SF gremlin- 1 concentrations and $\mathrm{KL}$ grade $(r=0.372, P<0.001$ and $r=0.483, P<0.001)$. Serum and SF gremlin- 1 concentrations and $\mathrm{KL}$ grades showed a positive association after multinomial logistic regression analysis $(P<0.001$ and $P<0.001)$.

\section{Discussion}

This study demonstrated the association of serum and SF gremlin-1 concentrations with $O A$. As we know, this is the first study indicating that serum and SF gremlin-1 concentrations were elevated in OA patients and that they were correlated with $\mathrm{OA}$ grading.

BMP, a major subgroup of the TGF- $\beta$ superfamily, could format and regenerate bone and cartilage [10]. BMP showed the anabolic response in cartilage explants and promoted chondroprogenitors cartilage matrix synthesis and endochondral bone 
Table 1. The parameters between case and control group.

\begin{tabular}{|c|c|c|c|}
\hline Characteristics & $\begin{array}{l}\text { knee OA patients } \\
\qquad(n=212)\end{array}$ & $\begin{array}{l}\text { healthy controls } \\
\qquad(n=125)\end{array}$ & $P$ value \\
\hline Age (years) & $62.68 \pm 8.67$ & $62.31 \pm 9.07$ & 0.712 \\
\hline Gender (male/female) & $90 / 122$ & $51 / 74$ & 0.766 \\
\hline BMI $\left(\mathrm{Kg} / \mathrm{m}^{2}\right)$ & $23.57 \pm 2.97$ & $23.89 \pm 3.23$ & 0.368 \\
\hline Serum gremlin-1 (ng/mL) & $475.08(385.24-548.61)$ & $389.31(297.00-473.85)$ & $<0.001$ \\
\hline SF gremlin-1 (ng/mL) & $296.94(256.93-365.48)$ & & \\
\hline
\end{tabular}

Table 2. Serum and SF gremlin-1 concentrations in different knee OA subgroups.

\begin{tabular}{|c|c|c|c|c|}
\hline $\begin{array}{c}\text { Gremlin-1 } \\
\text { (ng/mL) }\end{array}$ & $\begin{array}{c}\text { Grade } 2 \\
(n=64)\end{array}$ & $\begin{array}{c}\text { Grade } 3 \\
(n=91)\end{array}$ & $\begin{array}{c}\text { Grade } 4 \\
(n=57)\end{array}$ & $P$ value \\
\hline Serum & $435.40(339.04-498.35)^{b}$ & $481.95(398.58-533.19)^{a}$ & $533.19(456.40-596.96)^{\mathrm{a}, \mathrm{b}}$ & $<0.001$ \\
\hline SF & $267.65(221.50-313.02)^{b}$ & $320.39(257.28-367.09)^{a}$ & $376.53(290.01-424.27)^{a, b}$ & $<0.001$ \\
\hline
\end{tabular}

a $P<0.01$ vs. KL grade $2 ;{ }^{\text {b }} P<0.01$ vs. KL grade 3.

formation [11]. The expression of BMP was elevated in articular chondrocytes and the expression level was correlated with the severity of OA [12]. BMP receptor signaling played a key role in maintaining articular cartilage after birth [13]. The possible mechanism of BMP involved in OA pathogenesis is that BMPs regulated chondrocyte hypertrophy and matrix degradation [14].

BMP antagonists are a big family of structurally unrelated proteins. They serve as BMP antagonists by binding to BMP and then regulating the activities and functions of BMP [16]. Gremlin-1 is a typical BMP antagonist. Gremlin-1 has the ability to bind to BMP-2, BMP-4, and BMP-7. The equilibrium of BMP and its antagonists contributes to the homoeostasis of chondrocyte [16]. This balance is affected by inflammation, injury, or other stimuli. Then BMP was activated and led to chondrocyte hypertrophy, which is a potential mechanism of OA development. Furthermore, after the up-regulation of BMP, BMP antagonists were also up-regulated in OA cartilage. BMP antagonists could disturb activin/BMP activities and lead to decreased chondrocyte hypertrophy. This speculation was supported by a study by Tardif et al., which indicated that gremlin-1 expression was significantly elevated in OA chondrocytes and that BMP-2 and BMP-4 treatment significantly induced the expression of gremlin-1 in OA chondrocytes [8]. Treatment of gremlin-1 markedly inhibited terminal hypertrophic differentiation with no effects on the chondrogenesis [17]. This indicates the

\section{References:}

1. Felson DT: Clinical practice. Osteoarthritis of the knee. N Engl J Med, 2006; 354: 841-48 preventive role of gremlin-1 on hypertrophic differentiation of articular cartilage and OA development. Gremlin-1 expression was significantly elevated in early osteoarthritic cartilage, and increased slightly with disease progression [18]. Our results also demonstrated increased gremlin-1 concentrations in $\mathrm{OA}$ patients and a close correlation of gremlin-1 concentrations with $\mathrm{OA}$ disease progression. All these findings point to the therapeutic role of gremlin-1 in OA. In addition, other BMP antagonist family members such as follistatin and chordin also play a role in OA. Follistatin expression was significantly elevated in OA chondrocytes [8]. Chordin mRNA and protein levels were significantly down-regulated in osteoarthritic chondrocytes compared with normal cells [19]. This suggests the important role of BMP antagonist family in OA mechanism.

It should be mentioned that the current study inevitably has some limitations. First, this is a cross-sectional study. We may perform a prospective investigation to determine the exact role of gremlin-1 in OA. Second, this study was conducted in a relatively small sample.

\section{Conclusions}

Serum and SF gremlin-1 concentrations were positively correlated with the onset and severity of knee OA. 
3. Goldring MB, Otero $M$, Tsuchimochi $K$ et al: Defining the roles of inflammatory and anabolic cytokines in cartilage metabolism. Ann Rheum Dis, 2008; 67: iii75-82

4. Thijssen E, van Caam A, van der Kraan PM: Obesity and osteoarthritis, more than just wear and tear: Pivotal roles for inflamed adipose tissue and dyslipidaemia in obesity-induced osteoarthritis. Rheumatology (Oxford), 2015; 54: 588-600

5. Ducy P, Karsenty G. The family of bone morphogenetic proteins. Kidney Int, 2000; 57: 2207-14

6. Wellbrock J, Harbaum L, Stamm H et al: Intrinsic BMP antagonist Gremlin-1 as a novel circulating marker in pulmonary arterial hypertension. Lung, 2015; 193: 567-70

7. Blaney Davidson EN, Vitters EL et al: Elevated extracellular matrix production and degradation upon bone morphogenetic protein-2 (BMP-2) stimulation point toward a role for BMP-2 in cartilage repair and remodeling. Arthritis Res Ther, 2007; 9: R102

8. Tardif G, Hum D, Pelletier JP et al: Differential gene expression and regulation of the bone morphogenetic protein antagonists follistatin and gremlin in normal and osteoarthritic human chondrocytes and synovial fibroblasts. Arthritis Rheum, 2004; 50: 2521-30

9. Singh AK, Kalaivani M, Krishnan A et al: Prevalence of osteoarthritis of knee among elderly persons in urban slums using American College of Rheumatology (ACR) criteria. J Clin Diagn Res, 2014; 8: JC09-11

10. Minina E, Wenzel HM, Kreschel C et al: BMP and Ihh/PTHrP signaling interact to coordinate chondrocyte proliferation and differentiation. Development, 2001; 128: 4523-34
11. Benz K, Breit S, Lukoschek M et al: Molecular analysis of expansion, differentiation, and growth factor treatment of human chondrocytes identifies differentiation markers and growth-related genes. Biochem Biophys Res Commun, 2002; 293: 284-92

12. Nakase T, Miyaji T, Tomita T et al: Localization of bone morphogenetic protein-2 in human osteoarthritic cartilage and osteophyte. Osteoarthritis Cartilage, 2003; 11: 278-84

13. Rountree $\mathrm{RB}$, Schoor $\mathrm{M}$, Chen $\mathrm{H}$ et al: BMP receptor signaling is required for postnatal maintenance of articular cartilage. PLoS Biol, 2004; 2: e355

14. Papathanasiou I, Malizos KN, Tsezou A: Bone morphogenetic protein-2-induced Wnt/ $\beta$-catenin signaling pathway activation through enhanced lowdensity-lipoprotein receptor-related protein 5 catabolic activity contributes to hypertrophy in osteoarthriticchondrocytes. Arthritis Res Ther, 2012; 14: R82

15. Canalis E, Economides AN, Gazzerro E: Bone morphogenetic proteins, their antagonists, and the skeleton. Endocr Rev, 2003; 24: 218-35

16. Merino R, Rodriguez-Leon J, Macias D et al: The BMP antagonist Gremlin regulates outgrowth, chondrogenesis and programmed cell death in thedeveloping limb. Development, 1999; 126: 5515-22

17. Leijten JC, Emons J, Sticht $C$ et al: Gremlin 1, frizzled-related protein, and Dkk-1 are key regulators of human articular cartilage homeostasis. Arthritis Rheum, 2012; 64: 3302-12

18. Tardif G, Pelletier JP, Boileau C et al: The BMP antagonists follistatin and gremlin in normal and early osteoarthritic cartilage: An immunohistochemical study. Osteoarthritis Cartilage, 2009; 17: 263-70

19. Tardif G, Pelletier JP, Hum D et al: Differential regulation of the bone morphogenic protein antagonist chordin in human normal and osteoarthritic chondrocytes. Ann Rheum Dis, 2006; 65: 261-64 